\section{Application of an $X-Y$ recorder as a versatile mathematical instrument}

\section{F Graf and A Penzkofer}

Fachbereich Physik der Universität Regensburg, 8400 Regensburg, Germany

\section{Received 10 May 1979, in final form 13 July 1979}

Abstract An $X-Y$ recorder is used in connection with an on-line computer as a digitiser of graphical points and curves. Determinations of lengths, areas, integrals and derivatives are described.

\section{Introduction}

Mathematical instruments allow the analysis of graphical data. Various analogue devices have been introduced to display the coordinates of points (coordinatographs), to measure the lengths of curves (curvimeters), to determine the areas and momenta of figures (planimeters), to integrate curves (integrimeters, integraphs), to calculate Fourier components (harmonic analysers), and to differentiate plotted functions (derivators; for references see Willers 1943, 1971 and Meyer zur Capellen 1949). In the last few years electronic devices for the evaluation of graphics have been developed (electronic digitisers, electronic planimeters) and interfaced to digital computers (Cotter 1973, Fulton 1975, Gado et al 1975, Kamm 1977, Prytula 1977, Woodward 1977). In this paper we use an $X-Y$ recorder interfaced with CAMAC modules to a minicomputer to analyse graphical data.

\section{Operation principle}

The experimental layout of the graphics calculator system is shown in figure 1 . The graphical data sheet is loaded to the left $X-Y$ recorder (Hewlett Packard 7035 B). The writing pin of the plotter is manually positioned to the data points and guided along curves by adjusting the voltage at the $X$ and $Y$ inputs with two ten-turn potentiometers $P_{1}$ and $P_{2}$. A stable DC voltage supply is used (Gossen T1K30B). The voltages at the $X$ and $Y$ inputs are measured with an analoguedigital converter ADC (CAMAC module Ortec AD 811). This converter is gated with a pulse generator PG (Chronetics PG-10) and interfaced with a CAMAC crate controller (Dietz CC 621) to a minicomputer (Dietz 621). The data input to the converter starts by closing the switch $S_{2}$. When a gate

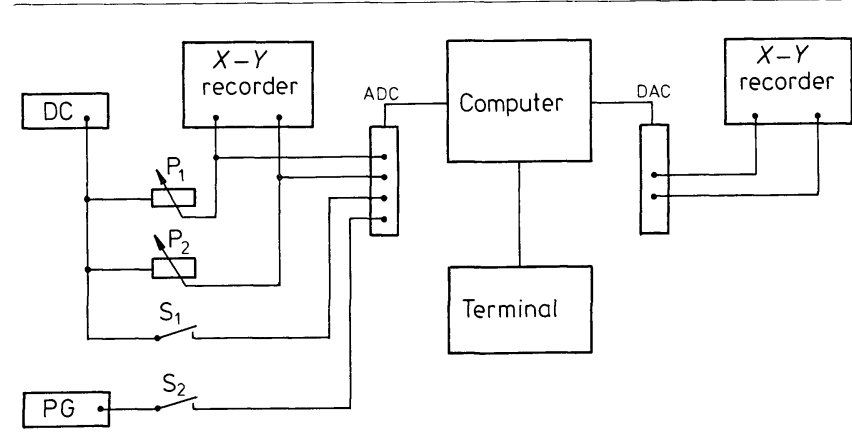

Figure 1 Experimental set-up. DC, stable DC voltage; PG, pulse generator; ADC, analogue-digital converter; DAC, digital-analogue converter, $\mathrm{P}_{1}$ and $\mathrm{P}_{2}$, ten-turn potentiometers. $S_{1}$, stop shutter; $S_{2}$, start and single gate shutter.

pulse is present at the analogue-digital converter the $X$ and $Y$ input voltages are fed to the registers of the converter and read by the computer. The repetition rate of the pulse generator determines the rate of data acquisition. Mathematical operations on the input data are carried out in real time. The results may be plotted by the $X-Y$ recorder on the right. This recorder is activated from the computer via a digital-analogue converter DAC (CAMAC module kinetic systems 3112 ). The data acquisition is finished when a voltage signal is entered into a third input channel of the ADC by the manually operated switch $S_{1}$. After final mathematical operations the result may be displayed on the computer terminal.

\section{Applications}

The described system transforms analogue data plots to digital values. All kinds of mathematical operations may be applied to the digitised pictures, diagrams or points such as summation, subtraction, multiplication, division, integration, differentiation, harmonic analysis and statistical processing. We applied the system to digitise points, to measure curve lengths, to determine areas of figures and to integrate and differentiate functions. The results of test measurements are summarised in table 1.

The pin of the reading recorder was replaced by a magnifier with a cross hair to increase the resolution. The accuracy of the $X$ and $Y$ positions is determined by the resolution of the analogue-digital converter, the stability of the regulated DC voltage and the linearity of the $X-Y$ recorder. When a data field of $20 \mathrm{~cm} \times 20 \mathrm{~cm}$ was used, a resolution of $\pm 0 \cdot 1 \mathrm{~mm}$ was obtained for the digitised $X$ and $Y$ positions.

The measurement of curve lengths was accomplished by guiding the pin of the reading recorder along the curve and by adding up the distances between the digitised points. As an

Table 1 Applications of $X-Y$ recorder system.

\begin{tabular}{|c|c|c|c|c|}
\hline Application & Example & Accuracy & Repetition rate & Comment \\
\hline Digitising & Point in $20 \mathrm{~cm} \times 20 \mathrm{~cm}$ & $\pm 0.1 \mathrm{~mm}$ & single & \\
\hline Curve length & Circle $5 \mathrm{~cm}$ diameter & $10^{-2}$ & $0 \cdot 4 \mathrm{~s}^{-1}$ & \\
\hline Area & Circle $5 \mathrm{~cm}$ diameter & $2 \times 10^{-3}$ & $50 \mathrm{~s}^{-1}$ & \\
\hline Integration & $\int_{0}^{\pi} \sin (x) \mathrm{d} x$ & $5 \times 10^{-3}$ & $50 \mathrm{~s}^{-1}$ & 1 unit $=5 \mathrm{~cm}$ \\
\hline Differentiation & $\mathrm{d} \sin (x) / \mathrm{d} x, 0 \leqslant x \leqslant \pi$ & $10^{-2}$ & $0.4 \mathrm{~s}^{-1}$ & 1 unit $=5 \mathrm{~cm}$ \\
\hline
\end{tabular}




\section{Apparatus and techniques}

example we determined the circumference of a circle of $5 \mathrm{~cm}$ diameter. An accuracy of $\Delta l / l \approx 10^{-2}$ was obtained $(\Delta l$ is the difference between the measured length $l$ and the exact value). In length measurements all errors resulting from incorrect positioning of the pin are added up. A slow data acquisition rate increases the accuracy.

The area of figures was determined by leading the reading pin along the envelope (start and stop point are the same) and calculating the integral

$$
A=\int \mathrm{d} x \mathrm{~d} y=\oint y \mathrm{~d} x \approx \sum_{i} y_{i} \Delta x_{i} .
$$

The area of a circle of $5 \mathrm{~cm}$ diameter was measured with an accuracy of $\Delta A \mid A \approx 2 \times 10^{-3}$. The applied calculation averages out small positioning errors and the accuracy is increased with data input rate. Momenta of areas $M_{n, m}=\int x^{n} y^{m} \mathrm{~d} x \mathrm{dy}$ $=(n+1)^{-1}(m+1)^{-1} \oint y^{m+1} \mathrm{~d}\left(x^{n+1}\right)$ (see Willers 1943) may be determined with a modified algorithm.

The integration of functions which are presented as curves is similar to the measurement of areas. Integrals $I\left(x^{\prime}\right)=$ $\int_{x_{0}}^{x^{\prime}} y(x) \mathrm{d} x$ may be plotted at the writing $X-Y$ recorder, while the results of definite integrals may be displayed on the computer terminal. As an example we determined the integral $I=\int_{0}^{\pi} \sin (x) \mathrm{d} x$ (see table 1$)$.

The differentiation of curves was approximated by calculating difference quotients $(\mathrm{d} y / \mathrm{d} x \approx \Delta y / \Delta x)$. The obtained derivatives were plotted at the writing $X-Y$ recorder. As an example the determination of $\mathrm{d} \sin (x) / \mathrm{d} x$ is present in table 1 .

\section{Conclusions}

The described graphics calculator system with an $X-Y$ recorder, an analogue-digital converter, a CAMAC interface system and a minicomputer is a very helpful instrument to analyse graphical data. Other interfacing of an $X-Y$ recorder to a calculator may be used as effectively. The system involves no additional costs when a computer and an $X-Y$ recorder are available in the laboratory.

\section{Acknowledgment}

The authors thank Professor M Maier for helpful discussions.

\section{References}

Cotter W L 1973 Coordinate digitiser system

US Patent No. 3732369

Fulton P 1975 Mapping and computers

Advances in Computers eds M Rubinoff and M C Yovits

(New York: Academic Press) vol. 13 pp 73-108

Gado P, Griger A and Turnezey T 1975 Use of a projectordigitiser-calculator combination for the interpretation of diffraction photographs

J. Appl. Crystallogr. 8 557-8

Kamm V C 1977 Electronic coordinate position digitising system

US Patent No. 4054746

Meyer zur Capellen W 1949

Mathematische Instrumente (Leipzig: Akademische

Verlagsgesellschaft)

Prytula R 1977 Touch sensitive digitiser

Proc. 5th Man-Computer Communications Conf., Calgary

May 1977 (Ottawa: National Research Council Canada)

pp 29-30

Willers F A 1943

Mathematische Instrumente (München: Oldenburg)
Willers F A 1971

Methoden der Praktischen Analysis (Berlin: Walter de Gruyter)

Woodward F I 1977 A device for translating chart recorder information into digital form

J. Phys. E: Sci. Instrum. 10 213-6 